\title{
PERSPECTIVA FUTURA DE TIEMPO Y AUTOEFICACIA: UN ANÁLISIS DE MEDIACIÓN (DUREZA PSICOLÓGICA, ESPERANZA Y VITALIDAD) EN MÉXICO Y EE.UU. d。
}

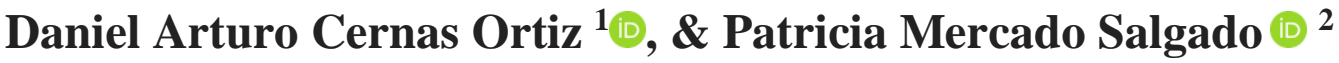 \\ Universidad Autónoma del Estado de México, Toluca de Lerdo, México.
}

\begin{abstract}
RESUMEN
Este trabajo tuvo por objetivo analizar la mediación de la dureza, la esperanza y la vitalidad en la relación entre la perspectiva futura de tiempo y la autoeficacia en el contexto laboral. También se analizó si tal mediación varía entre México y EE.UU. Se encuestó a un total de 583 estudiantes de Máster en Administración de los dos países. Se empleó el comando PROCESS (Hayes, 2013) en SPSS® (20) para analizar los datos. Los hallazgos sugieren que (en orden de poder de mediación) la esperanza, la dureza y la vitalidad transmiten casi todo el efecto de la perspectiva futura de tiempo en la autoeficacia y que los efectos mediadores son invariantes entre los países analizados.
\end{abstract}

Palabras Claves

autoeficacia; perspectiva de tiempo; diferencias culturales; dureza psicológica; esperanza

\begin{abstract}
This study's objective was to analyze the mediating effects of hardiness, hope, and vitality in the relationship between future time perspective and self-efficacy in the work context. We also analyzed whether such mediation effects vary between Mexico and the U.S. A total of 583 MBA students were surveyed in both countries. The PROCESS (Hayes, 2013) command in SPSS $®$ (20) was employed to analyze the data. The results suggest that (in order of mediating power) hope, hardiness, and vitality transmit almost all the future time perspective' effects to self-efficacy and that such mediating effects are invariant between the analyzed countries.
\end{abstract}

Keywords

self-efficacy; time perspective; cultural differences; hardiness; hope

\footnotetext{
1 Correspondence about this article should be addressed to Daniel Arturo Cernas Ortiz: $\underline{\text { dacernaso@uaemex.mx }}$

2 Conflicts of Interest: The authors declare that the research was conducted in the absence of any commercial or financial relationships that could be construed as a potential conflict of interest.
} 


\section{FUTURE TIME PERSPECTIVE AND SELF-EFFICACY: A MEDIATION ANALYSIS (PSYCHOLOGICAL HARDINESS, HOPE, AND VITALITY) IN MEXICO AND THE U.S.A.}

\section{Introducción}

La teoría social cognitiva indica que la autoeficacia es un término clave para entender la motivación y el éxito. La autoeficacia se define como la creencia, o convicción, de que se puede ejecutar un comportamiento dado y alcanzar un cierto nivel de desempeño en una situación determinada (Bandura, 1977). En el trabajo, las personas con fuertes creencias en su eficacia personal tienden a ponerse (o aceptar) retos altos y a perseverar en ellos hasta alcanzarlos (Locke \& Latham, 2009). Por ello, no es de sorprender que, los empleados con una autoeficacia sólida son aquellos que suelen dar mejores resultados (Miraglia, Cenciotti, Alesandri \& Borgogni, 2017). La autoeficacia, sin embargo, no surge en el vacío, sino que tiene como fuente principal a las experiencias directas de éxito (enactive mastery) (Bandura, 1977; Oettingen, 1995). Es decir, la gente llegamos a creer que podemos hacer algo después de intentarlo y de lograrlo (Bandura, 1977). Ser exitoso, por supuesto, propicia el establecimiento de metas más retadoras, lo que eleva la motivación y el desempeño (Bandura \& Locke, 2003).

La perspectiva futura de tiempo (PFT) también es importante para comprender la perseverancia y el éxito. En general, la perspectiva de tiempo es un sesgo cognitivo que ayuda a interpretar la realidad en una forma consistente con él y que se comporta como un rasgo (o disposición) personal relativamente estable que influencia los juicios, las emociones y las acciones (Zimbardo \& Boyd, 1999). En particular, la PFT se refiere a la tendencia a pensar en el mañana y a tomar en cuenta las consecuencias futuras de las acciones presentes (Stolarski, Mattews, Postek, Zimbardo \& Bitner, 2014). Por ello, no es sorprendente que la PFT se relacione positivamente con fenómenos tales como el cuidado del agua (Corral-Verdugo, Fraijo-Sing \& Pinheiro, 2006). En el trabajo, la PFT es importante ya que, por ejemplo, mediante proyecciones del futuro es que los jefes y sus subordinados establecen objetivos y formulan programas y estrategias para lograrlos (Cernas-Ortiz, Mercado-Salgado y Davis, 2018). Dado que la PFT se caracteriza por el establecimiento de metas, y que se relaciona positivamente con la meticulosidad y el interés en recompensas (Zimbardo \& Boyd, 1999), es que la PFT puede fomentar un alto desempeño laboral. Es de notar que la PFT presupone firmes pensamientos de agencia 
(creer que uno sí puede crear y/o modificar su destino) ya que de otra forma no se entendería la intención de articular el futuro por medio de objetivos y metas.

Investigaciones anteriores indican que la PFT y la autoeficacia están relacionadas (e.g. Epel, Bandura \& Zimbardo, 1999; Park \& Jung, 2015). La PFT predispone a trabajar duro para alcanzar las metas postergando los placeres inmediatos (Zimbardo y Boyd, 1999) y promueve la construcción de capacidades necesarias para lograr objetivos con un alto desempeño (Walker \& Tracey, 2012). La PFT, por lo tanto, favorece el desarrollo de las experiencias directas de éxito contribuyendo al desarrollo de la autoeficacia (CernasOrtiz et al., 2018). Los estudios previos, sin embargo, suponen que la relación entre la PFT y la autoeficacia es directa. Aunque esta suposición es plausible, resulta un tanto problemática tomando en cuenta que la relación entre la PFT y la autoeficacia podría estar mediada por otras variables que tanto promueven las experiencias directas de éxito, como también están influenciadas por la PFT.

De particular relevancia resulta examinar si la relación entre la PFT y la autoeficacia podría estar mediada por factores que, con una clara orientación al futuro, estimulan la resiliencia y mantienen la perseverancia y el entusiasmo ante resultados adversos. Es bien sabido que entre más retadora es una meta, más es la probabilidad de fracasar al intentar alcanzarla. Algunas personas se dan por vencidas ante la adversidad y no perseveran, pero otras sí lo hacen hasta lograr lo que se proponen (Seligman, 2006). Como resultado, mientras que las personas que perseveran y tienen éxito desarrollarían una autoeficacia sólida, las que no lo hacen tenderían más a formarse creencias de incompetencia que de autoeficacia (Cernas-Ortiz et al., 2018). Si la capacidad para sobreponerse al fracaso es clave para la formación de la autoeficacia, y tal capacidad nace de firmes pensamientos de agencia (Epel et al., 1999), entonces tales factores como la dureza (hardiness), la esperanza (hope) y la vitalidad (vitality) mediarían la relación entre la PFT y la autoeficacia. Como se explica a continuación, estos factores promueven la perseverancia y claramente suponen pensamientos de agencia directamente asociados con la PFT. La dureza se ha identificado como un antecedente importante del alto desempeño y la salud en condiciones de gran estrés (Maddi, 2013). La esperanza y la vitalidad son, de entre un total de 24 fortalezas del carácter, las que más impacto tienen en el bienestar subjetivo (Park, Peterson y Seligman, 2004). 


\section{El papel mediador de la dureza, la esperanza y la vitalidad}

La dureza fomenta respuestas saludables a las constantes presiones de la vida diaria (Maddi, 2002) promoviendo el bienestar subjetivo (Skomorovsky \& Sudom, 2011), o lo que es lo mismo, la felicidad. La dureza es una disposición individual que, como constructo, se compone de tres actitudes relacionadas: Compromiso (convicción de que uno debe permanecer involucrado no importando qué tal mal se pongan las cosas), control (creer que uno puede tornar un desastre en algo positivo) y reto (asumir que la vida es estresante y que los problemas son desafíos a superar para lograr el éxito) (Maddi, 2013). Una persona dura (psicológicamente hablando) es aquélla que percibe el mundo como menos amenazante, que se mantiene motivada ante el fracaso debido a que lo concibe como una oportunidad de aprendizaje, y que está convencida de que tarde de o temprano logrará lo que se propone (Cole, Feild \& Harris, 2004). Por supuesto, una persona con estas características es más posible que desarrolle un fuerte sentido de autoeficacia ya que no se amedrenta ante la retroalimentación negativa (al contrario, aprende) y persevera hasta lograr sus metas (lo que contribuye a las experiencias directas de éxito). Por otra parte, la dureza estaría fuertemente influenciada por la PFT debido a que es un concepto claramente orientado a persistir hasta lograr algo (Maddi, 2013), y esto refleja fuertes pensamientos de agencia orientados al futuro. Si la PFT influencia a la dureza y ésta, a su vez, a la autoeficacia, entonces la dureza mediaría entre la PFT y la autoeficacia.

La esperanza es una fortaleza del carácter que predice el desempeño, la salud y el ajuste psicológico (Snyder, Rand, King, Feldman \& Woodward, 2002). Este factor se define como el estado motivacional basado en un sentido derivado en forma interactiva de que las metas se pueden lograr (agencia) y de que existen varias formas de hacerlo (planeación) (Snyder, Sympson, Ybasco, Borders, Babyak \& Higgins, 1996). La esperanza involucra un patrón cognitivo por el cual el fracaso se atribuye a causas que pueden cambiar en el futuro, como el esfuerzo (cuya intensidad es controlable por la persona) o incluso la mala suerte (Weiner, 2010). La esperanza, por lo tanto, mantiene la persistencia ya que la expectativa de lograr resultados positivos en la vida se sostiene a pesar de los infortunios (Park, et al., 2004). Como se infiere, un fuerte sentido de esperanza fomenta, eventualmente, el logro de objetivos y esto contribuye a las experiencias directas de éxito que dan origen a la autoeficacia. Si, por otro lado, la esperanza está fuertemente influenciada por factores que propician, además de la planeación, el sentido de agencia (Snyder et al., 2002), entonces la PFT estaría 
fuertemente relacionada con ella (Cernas-Ortiz et al., 2018). En corto, la relación entre la PFT y la autoeficacia puede estar mediada por la esperanza debido a que la PFT influenciaría a la esperanza y ésta a la autoeficacia.

La vitalidad es un rasgo personal relativamente estable asociado al bienestar subjetivo (Peterson, Park, Hall \& Seligman, 2009) que se refiere a la experiencia consciente de poseer energía y vivacidad (Rayan \& Fredrerick, 1997). En las organizaciones, las personas de gran vitalidad son más propensas a experimentar vigor (Peterson et al., 2009). Debido a que el vigor está asociado al logro de objetivos y al alto desempeño (Yongxing, Hongfei, Baoguo \& Lei, 2017), la vitalidad puede relacionarse con la autoeficacia ya que promueve experiencias directas de éxito. La vitalidad también promueve la persistencia ante resultados adversos ya que tiene origen en la experiencia subjetiva de poder se causa de algo (cuando la energía viene de uno mismo es que se siente más vivo; Rayan \& Fredrerick, 1997). Percibirse como la causa de algo contribuye a la idea de que las cosas pueden cambiar y mejorar (p.ej. realizando más esfuerzo) y esto mantiene la persistencia. Tales pensamientos de agencia relacionan a la vitalidad con la PFT. En otras palabras, si la PFT se caracteriza por estructurar el futuro mediante metas y trabajar duro para lograrlas (Stolarski et al., 2014), entonces la PFT incitaría la vitalidad pues es una fuente subjetiva de energía y vigor. Zimbardo y Boyd (1999) encuentran una fuerte correlación positiva entre la PFT y la energía, así como una fuerte correlación negativa entre la PFT y la depresión (que implica pasividad y fatiga). Si la PFT puede influenciar a la vitalidad y ésta a la autoeficacia, entonces la vitalidad mediaría entre la PFT y la autoeficacia.

\section{El rol moderador de la cultura nacional}

No obstante, la cultura nacional (los valores, normas y suposiciones compartidos por un grupo social, o el "software de la mente"; Hofstede, 2015) podría alterar (moderar) el efecto mediador de la dureza, la esperanza y la vitalidad en la relación entre la PFT y la autoeficacia. Las diferencias culturales entre México y Estados Unidos (EE.UU.) son un contexto apropiado para explorar esta idea. Hofstede, Hofstede y Minkov (2010) encontraron que ambos países difieren en seis dimensiones culturales: masculinidadfeminidad, evasión de la incertidumbre, distancia de poder, individualismo-colectivismo, corto versus largo plazo e indulgencia versus restricción. Los resultados de Hofstede y sus colegas indican que México es más masculino, más tendiente a evadir la 
incertidumbre, con mayor distancia de poder, menos individualista, más orientado al corto plazo y más indulgente que EE.UU. Estas diferencias, por supuesto, pueden hacer que los efectos mediadores sugeridos en este documento varíen entre ambos países.

Para ejemplificar un poco lo anterior, tómese en cuenta que la cultura podría influenciar tanto el tipo de información que la mente toma como base para formarse ideas de autoeficacia, como la selección de esa información y su ponderación e integración al momento de juzgar la eficacia personal para realizar una tarea (Oettingen, 1995). De tal suerte, en sociedades altamente individualistas (egocéntricas), las experiencias directas de éxito contribuirían más a la formación de la autoeficacia que en sociedades menos individualistas (colectivistas o sociocéntricas) (Oettingen, 1995). Esto, debido a que mientras que en culturas individualistas el logro individual es algo que se espera, se valora y que tiene gran significado (Seligman, 2006), en sociedades colectivistas los juicios sobre el éxito personal se basan más en las opiniones que los miembros del grupo tienen de las capacidades de la persona, que en lo que ella misma piensa de sus logros (Oettingen, 1995). Por ello, el efecto que la PFT pueda tener en la autoeficacia a través de la dureza, la esperanza y la vitalidad puede variar debido (por ejemplo) a las diferencias en individualismo entre la cultura Mexicana y la de EE.UU. identificadas por Hofstede et al. (2010).

\section{Este trabajo}

Con base en los antecedentes anteriores, el propósito del presente estudio es poner a prueba las siguientes hipótesis:

H 1. La dureza media la relación entre la PFT y la autoeficacia.

H 2. La esperanza media la relación entre la PFT y la autoeficacia.

H 3. La vitalidad media la relación entre la PFT y la autoeficacia.

H 4. La cultura nacional (México versus EE.UU.) modera el efecto mediador de la dureza, la esperanza y la vitalidad en la relación entre la PFT y la autoeficacia.

La Figura 1 ilustra gráficamente las relaciones indicadas por las hipótesis anteriores. En esta figura, la línea punteada representa al efecto directo (ya documentado en el pasado) que puede haber entre PFT y autoeficacia. 


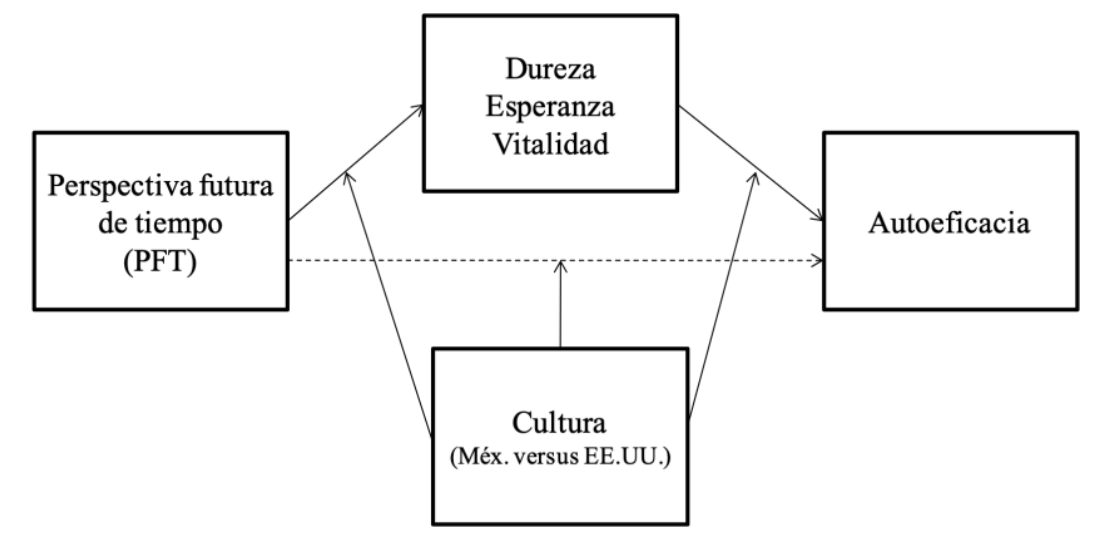

Figura 1. Modelo de investigación

Para lograr el propósito de este trabajo se utiliza el comando PROCESS (Hayes, 2013) en SPSS ${ }^{\circ}(20)$, ya que permite analizar paralelamente el efecto mediador de un conjunto de variables en la relación entre otras dos. Este comando suma los efectos de todas las variables mediadoras para determinar un efecto indirecto (mediador) total y comparar los efectos indirectos individuales determinando la variable que más contribuye al efecto indirecto total (y al total). De esta forma, no sólo se puede determinar cuánto se reduce el efecto total de una variable sobre otra cuando se toman en cuenta todos los mediadores juntos (y por lo tanto determinar el efecto directo neto), sino que se puede establecer si los efectos mediadores son estadísticamente diferentes entre ellos. PROCESS también ayuda a determinar los efectos mediadores a los diferentes valores de una variable moderadora y a saber si entre ellos son significativamente distintos.

En lo principal, este trabajo contribuye a entender mejor los matices de la relación entre la temporalidad psicológica y la autoeficacia. El desarrollo de la autoeficacia no es atemporal ya que, por ejemplo, un individuo puede (o no) persistir en sus experiencias directas de éxito debido a su preocupación por el futuro y a sus expectativas con respecto a él (o bien, debido a sus experiencias pasadas o inclinaciones presentes). El tiempo es importante porque, ya sea que se conciba como una propiedad subjetiva de la conciencia personal, o como un atributo objetivo de eventos y episodios, la conducta existe en y a través del tiempo (Navarro, Roe \& Artiles, 2015).

\section{Método}

\section{Participantes}

Un total de 583 estudiantes de Máster en Administración ( $M B A$ en inglés) participaron en el estudio (Méx. = 299, EE.UU. = 284). De esta cantidad, 22 respuestas 
(3.8\%) se descartaron debido a información incompleta o incorrecta. La base de datos final se compuso de 561 observaciones (Méx. = 287, EE.UU. =274). La edad promedio fue de 30.35 años (Méx. = 31.32, EE.UU. = 29.34) con una desviación estándar de 6.6 (Méx. = 5.77, EE.UU. = 7.24). La gran mayoría de los participantes tenía un empleo al momento en que se realizó el estudio (Méx. = 268, EE.UU. = 230).

Los respondientes fueron alumnos regulares de una universidad en el centro de México y una universidad en el suroeste de EE.UU.

\section{Procedimiento}

Se empleó una encuesta autoadministrada (autoinformada) aplicada a los estudiantes durante sus horas de clase. La participación en el estudio se pidió verbalmente siendo el llenado del cuestionario completamente voluntario. En todo momento se preservó el anonimato y se comunicó que no había respuestas buenas ni malas y que los datos serían analizados en el agregado. También se dijo a los estudiantes que ellos podían dejar de contestar el cuestionario en el momento en que así lo quisieran. A los estudiantes desempleados se les pidió que contestaran con base en su más reciente experiencia laboral.

Para la aplicación de la encuesta en México, todas las preguntas fueron traducidas al español (versión mexicana) de su original en inglés. No se utilizaron traducciones literales a menos que la traducción en español fuera directamente equivalente a la versión inglesa. En una primera etapa, todos los reactivos se pasaron al español por una persona no involucrada en la investigación. Luego, las versiones españolas se retradujeron al inglés por otro individuo. Las dos versiones en inglés (la original y la retraducida) se compararon y se detectaron discrepancias, mismas que fueron discutidas y corregidas.

\section{Instrumentos}

$P F T$. Ésta se midió mediante la escala de 13 reactivos de PFT contenida en el Inventario de Perspectiva de Tiempo de Zimbardo y Boyd (1999). Los reactivos se presentaron en una escala Likert de cinco graduaciones (para nada característico de mí muy característico de mí). La oración "Cuando quiero lograr algo, establezco metas y tomo en cuenta los medios para alcanzarlas" es un ejemplo de la escala.

Autoeficacia. Para medirla se utilizó la forma corta de la escala de autoeficacia ocupacional de Schyns y von Collani (2002) que consiste en ocho reactivos que capturan 
diferencias en autoeficacia a través de varias profesiones y trabajos. Esta escala es la más apropiada para este estudio debido a que la muestra utilizada contiene personas de diferentes profesiones y empleos. Otras escalas de autoeficacia son específicas para tareas concretas. Los reactivos se presentaron en una escala Likert de siete graduaciones (para nada cierto - muy cierto). El enunciado "Me siento preparado(a) para afrontar la mayoría de las exigencias de mi trabajo" ejemplifica la escala.

Dureza. Se utilizó la escala de Cole, Bruch y Vogel (2006) para medir esta variable. La escala tiene seis reactivos en un formato Likert de siete graduaciones (nunca - siempre). "A pesar de algunos reveces, permanezco comprometido(a) para lograr mis labores de trabajo" es una muestra de la escala.

Esperanza. Ésta se midió por medio de la escala de Snyder et al. (1996) que contiene seis reactivos en un formato Likert de siete graduaciones (para nada cierto - muy cierto). "Si estuviera en un aprieto, podría pensar en muchas formas para salir de él" es un ejemplo de la escala.

Vitalidad. La escala de Ryan y Frederick (1997) se utilizó para medir esta variable que contiene siete reactivos en formato Likert de siete graduaciones (para nada cierto muy cierto). La oración "Espero impaciente el nuevo día” ejemplifica la escala.

Antes de analizar los datos, todas las escalas fueron deculturizadas siguiendo las instrucciones de Bond (1988). Esto es, los reactivos fueron estandarizados para cada cultura en forma independiente. Este procedimiento no altera las relaciones entre los constructos, pero sí equipara la distribución de las respuestas entre las muestras removiendo influencias culturales en el uso de las escalas.

\section{Análisis de datos}

Validez. Ésta se probó mediante un análisis factorial confirmatorio (AFC en LISREL® 8.8) que involucró la comparación de dos modelos para cada país en forma separada. En el primer modelo se forzó a todos los reactivos a cargar en un solo constructo latente. En el segundo modelo los reactivos se asignaron al constructo al que teóricamente pertenecían (PFT, dureza, esperanza, vitalidad y autoeficacia). Mediante este método se encuentra evidencia de validez estructural si entre la solución de un factor y la de cinco existen diferencias significativas en términos de $\Delta \chi^{2} ; \mathrm{y}$, si la solución de cinco factores muestra índices de bondad de ajuste superiores a la de uno. Cuando la estructura de covarianza observada refleja la estructura de covarianza teórica de los datos, la solución 
en la que cada reactivo es asignado al constructo teórico al que supuestamente pertenece debe ser superior a una solución en la que no hay diferenciación de constructos (Hair, et al., 2006).

Adicionalmente, se realizaron pruebas de invarianza configural y métrica involucrando la comparación directa de los modelos de cinco factores de ambas muestras. Esto se hizo para determinar que los cinco factores investigados existiesen paralelamente en las muestras de los dos países y comprobar que las graduaciones de las escalas de medición se utilizaron de forma similar en ambos (Hair, et al., 2006).

Descriptivos. Se calculó la media y la desviación estándar de todas las variables para tener una idea general de los datos. Se calcularon correlaciones bivariadas entre los constructos como paso preliminar a la prueba de las hipótesis.

Varianza del método común. Este potencial sesgo de medición se probó mediante el procedimiento sugerido por Lindell y Whitney (2001).

Prueba de hipótesis. Las hipótesis se probaron mediante el comando PROCESS (Hayes, 2013) en SPSS $®(20)$.

\section{Resultados}

Validez. En la Tabla 1 se observa la comparación de los modelos de uno y cinco factores para México y EE.UU. En esta tabla también se muestran las pruebas de invarianza configural y métrica. Como se muestra, la prueba de diferencias significativas entre los modelos de uno y cinco factores $\left(\Delta \chi^{2}\right)$ indica que los modelos difieren significativamente. Además, tanto para México como para EE.UU., el modelo (solución) de cinco factores muestra índices de bondad de ajuste superiores y en concordancia con los valores usuales de ajuste apropiado (Kilne, 2011). Esto sugiere que, en los países analizados, los reactivos de las escalas se agrupan (convergen) adecuadamente en cinco factores que se discriminan adecuadamente entre sí.

En la Tabla 1 se muestra que cuando las dos muestras se fuerzan a ser equivalentes en términos configurales, los índices de bondad de ajuste son adecuados. Asimismo, los índices de bondad de ajuste permanecen casi inalterados cuando las cargas factoriales se fuerzan a ser equivalentes entre los países. Puesto que la diferencia en ajuste entre los modelos configural y métrico es significativa, se puede decir que, en las dos muestras, no sólo existen cinco factores equivalentes, sino que, una vez estandarizados los datos, las escalas de medición muestran un rango de respuesta similar en los dos países. 
Tabla 1.

Validez estructural e invarianza de las escalas de medición

\begin{tabular}{|c|c|c|c|c|c|c|c|c|c|}
\hline Modelo / Indicador & $\chi^{2}$ & gl & RMSEA & CFI & NNFI & $\chi^{2 / g 1}$ & $\Delta \chi^{2}$ & & $\Delta \mathrm{CFI}$ \\
\hline México: Solución de un factor & $3060.4 * *$ & 740 & 0.11 & 0.88 & 0.87 & 4.14 & & & \\
\hline México: Solución de cinco factores & $1334.06 * *$ & 723 & 0.05 & 0.96 & 0.95 & 1.85 & 1726.3 & $* *$ & 0.08 \\
\hline EE.UU.: Solución de un factor & $3368.51 * *$ & 740 & 0.11 & 0.92 & 0.91 & 4.55 & & & \\
\hline EE.UU.: Solución de cinco factores & $1264.52 * *$ & 723 & 0.05 & 0.97 & 0.97 & 1.75 & 2104 & $* *$ & 0.05 \\
\hline $\begin{array}{l}\text { Modelo de equivalencia de estructura } \\
\text { factorial (invarianza configural) }\end{array}$ & $3061.08 * * *$ & 1460 & 0.06 & 0.96 & 0.95 & 2.10 & & & \\
\hline $\begin{array}{l}\text { Modelo de equivalencia de carga } \\
\text { factorial (invarianza métrica) }\end{array}$ & $3189.79 * * *$ & 1500 & 0.06 & 0.96 & 0.95 & 2.13 & 128.71 & $* *$ & 0.0 \\
\hline
\end{tabular}

Descriptivos. En la Tabla 2 la confiabilidad $(\alpha)$ y las correlaciones se calculan con datos estandarizados para la muestra combinada total. Utilizando datos no estandarizados, los estadísticos descriptivos se muestran para la muestra combinada total y para cada muestra independiente. Como se observa, la confiabilidad es aceptable en todos los casos. También, mientras que la media de PFT es mayor en EE.UU., la media de las otras variables es mayor en México. Las diferencias de medias entre los países son significativas, aunque este resultado no es confiable debido a que las diferentes aproximaciones culturales al uso de las escalas pueden explicarlas. Las correlaciones bivariadas son moderadas y en concordancia con lo encontrado en otras investigaciones (p.ej. Cernas-Ortiz et al., 2018).

Tabla 2.

Descriptivos y correlaciones

\begin{tabular}{|c|c|c|c|c|c|c|c|c|c|c|c|c|c|}
\hline \multirow{2}{*}{ Variable } & \multirow{2}{*}{$\alpha$} & \multicolumn{3}{|c|}{ Media } & \multirow{2}{*}{\multicolumn{2}{|c|}{$t$}} & \multicolumn{3}{|c|}{ Desviación estándar } & \multirow{2}{*}{1} & \multirow{2}{*}{2} & \multirow{2}{*}{3} & \multirow{2}{*}{4} \\
\hline & & General & México & EE.UU. & & & General & México & EE.UU. & & & & \\
\hline $1 \mathrm{PFT}$ & 0.73 & 3.75 & 3.70 & 3.80 & -2.34 & * & 0.51 & 0.50 & 0.52 & & & & \\
\hline 2 Dureza & 0.83 & 6.00 & 6.19 & 5.81 & 5.87 & $* *$ & 0.77 & 0.63 & 0.85 & $0.27 * *$ & & & \\
\hline 3 Esperanza & 0.85 & 5.67 & 5.91 & 5.42 & 6.07 & $* *$ & 0.97 & 0.89 & 0.99 & $0.35 * *$ & $0.58 * *$ & & \\
\hline 4 Vitalidad & 0.82 & 5.18 & 5.41 & 4.93 & 5.83 & $* *$ & 0.99 & 0.83 & 1.08 & $0.26^{* *}$ & $0.46 * *$ & $0.56^{* *}$ & \\
\hline 5 Autoeficacia & 0.91 & 5.77 & 5.94 & 5.60 & 4.41 & $* *$ & 0.92 & 0.80 & 1.01 & $0.31 * *$ & $0.73 * *$ & $0.72 * *$ & $0.51 * *$ \\
\hline
\end{tabular}

Varianza del método común. Una vez controlado este fenómeno en las correlaciones de la Tabla 2, éstas variaron muy poco (.03) manteniendo su nivel de significatividad. Por ello, la prueba de las hipótesis no está contaminada seriamente por sesgos de medición.

Prueba de hipótesis. Las hipótesis 1, 2 y 3 (respectivamente) indican que la dureza, la esperanza y la vitalidad median la relación entre la PFT y la autoeficacia. La Tabla 3 muestra los resultados de la prueba de mediación. En primer lugar, se observa que el efecto total de la PFT en la autoeficacia es positivo y significativo y que, cuando 
se descompone, el efecto directo es muy pequeño y no significativo trasmitiéndose la mayor parte del efecto total indirectamente a través de la dureza, la esperanza y la vitalidad en conjunto. De hecho, la Tabla 3 indica que el 95\% del efecto total está mediado por las variables indicadas. En la misma tabla se muestra que todas las variables mediadoras presentan coeficientes significativos y que la que más media la relación entre la PFT y la autoeficacia es la esperanza (49\% del efecto total), seguida de la dureza (39\% del efecto total) y la vitalidad (7\% del efecto total). En conjunto, esta evidencia apoya las hipótesis 1,2 y 3.

Adicionalmente, tómese en cuenta que la diferencia en poder de mediación entre la dureza y la esperanza no es significativa $(-0.03, \mathrm{p}>0.05)$, pero sí entre la dureza y la vitalidad $(0.10, \mathrm{p}<0.05)$ y entre la esperanza y la vitalidad $(0.13, \mathrm{p}<0.05)$. Esto es, aunque la esperanza media más la relación entre la PFT y la autoeficacia que la dureza, la diferencia podría ser azarosa. Sin embargo, la dureza y la esperanza sí median más la relación en cuestión que la vitalidad y las diferencias sí es altamente probable que sean sistemáticas.

La Hipótesis 4 indica que la cultura nacional (México versus EE.UU.) modera la mediación de la dureza, la esperanza y la vitalidad en la relación en entre la PFT y la autoeficacia. La Tabla 4 muestra por separado los efectos mediadores de las variables en cuestión para México y para EE.UU. Como se observa, mientras que los efectos indirectos de la PFT en la autoeficacia a través de la dureza y la esperanza son significativos para ambos países, la diferencia entre coeficientes no es significativa. Con respecto a la vitalidad, se observa que los efectos indirectos a través de esta variable no son significativos en ningún país (lo que se puede explicar por la pérdida de poder estadístico en el análisis por muestras individuales) y que no hay diferencia significativa entre ellos. En conjunto, esta evidencia rechaza la hipótesis 4.

Como mera ilustración, la Figura 2 sugiere que los efectos indirectos de la PFT en la autoeficacia a través de la esperanza y la vitalidad son más fuertes en México. También, el efecto directo de la PFT en la autoeficacia y el efecto indirecto a través de la dureza serían más fuertes en EE.UU.

Es de notar que la prueba de las hipótesis incluyó a la edad (estandarizada por cada cultura), el sexo $(1=$ hombre, $2=$ mujer $)$ y el estatus laboral $(0=$ desempleado, $1=$ empleado) de los participantes como variables control. 
Tabla 3.

Sumario de los resultados de mediación

Efectos

Coeficientes y porcentajes

Efecto total de la PFT en la autoeficacia

Efecto directo de la PFT en la autoeficacia

Proporción del efecto total directo de la PFT

$5 \%$

Efecto indirecto total de la PFT en la autoeficacia a través de la

0.31

dureza, la esperanza y la vitalidad

Proporción del efecto total que está mediada

$95 \%$

Efecto indirecto a través de la dureza

0.13

Proporción del efecto total que está mediada por la dureza $39 \%$

0.16

Efecto indirecto a través de la esperanza

Proporción del efecto total que está mediada por la esperanza $49 \%$

Efecto indirecto a través de la vitalidad 0.02

Proporción del efecto total que está mediada por la vitalidad $7 \%$

Nota: Los números entre paréntesis son el error estándar $*=\mathrm{p}<0.05 ; * *=\mathrm{p}<0.01$

\section{Discusión}

La autoeficacia tiene como fuente principal a las experiencias directas de éxito, mismas que, a su vez, tienen distintos antecedentes. Entre ellos, algunos ampliamente estudiados como la meticulosidad (consietiousness) (Stajkovic, Bandura, Locke, Lee \& Sergent, 2018) y otros con atención emergente en la investigación como la PFT (Park \& Jung, 2015). En este trabajo se examinó el grado con el cual factores que estimulan la perseverancia ante la adversidad y promueven el éxito como la dureza, la esperanza y la vitalidad median la relación entre la PFT y la autoeficacia. 
Tabla 4.

Sumario de resultados de la mediación moderada

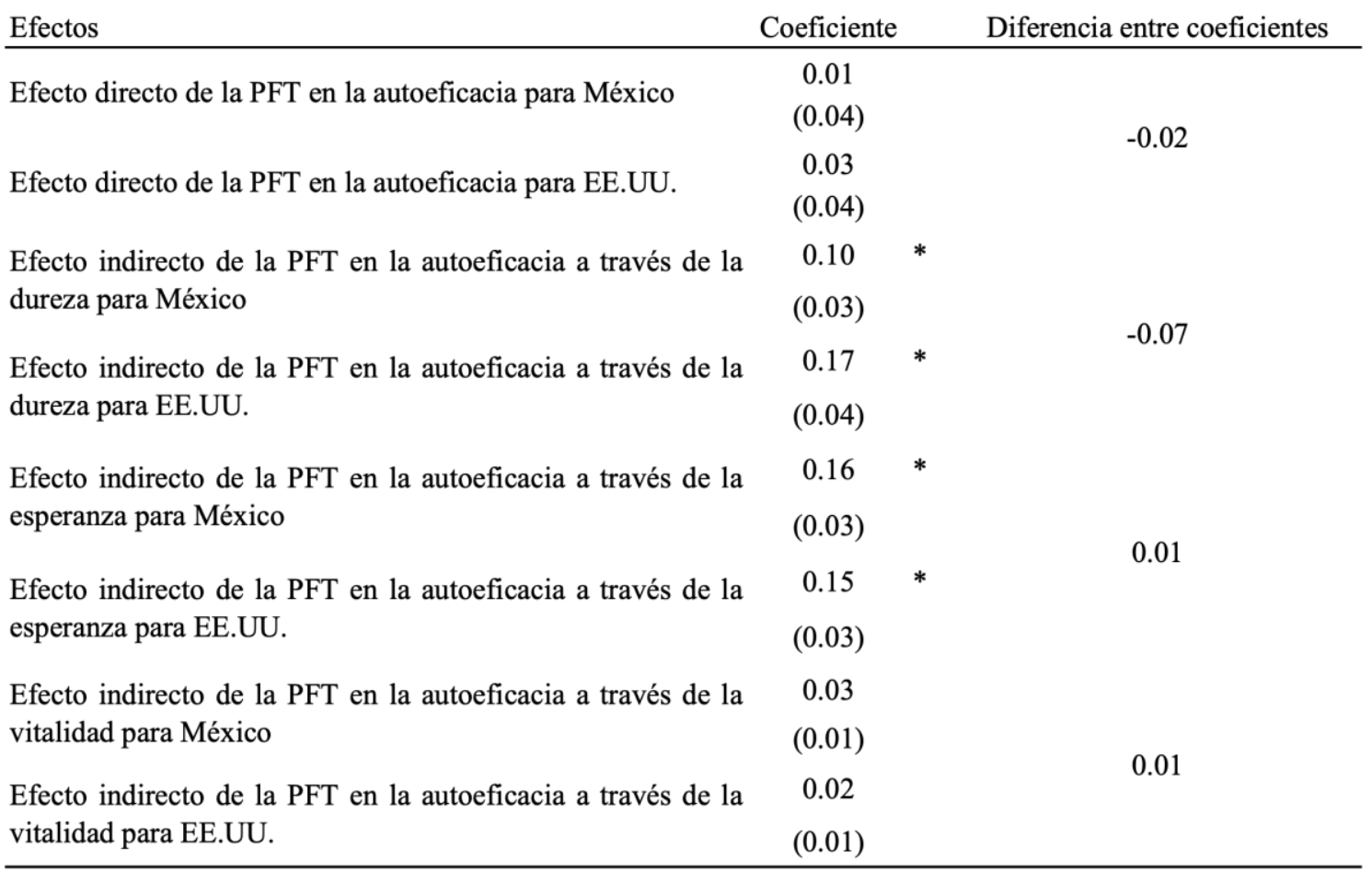

Nota: Los números entre paréntesis son el error estándar

$*=\mathrm{p}<0.05$

Dicho lo anterior, los hallazgos de este trabajo extienden el conocimiento actual dejando claro que la relación entre la PFT y la autoeficacia es mucho más matizada de lo que una relación directa entre ambas supone. Tal es así, que el efecto directo de la PFT en la autoeficacia es muy pequeño y no significativo una vez que se separa el efecto indirecto de los mediadores. A su vez, los mediadores analizados muestran diferente poder de mediación a grado tal que mientras que la esperanza sola media virtualmente la mitad del efecto total de una variable sobre la otra, la vitalidad lo hace en una forma bastante reducida. Tal parece que la PFT tiene efectos distantes en la autoeficacia que necesitan trasmitirse por otros factores que, aunque asociados a la PFT, sean más cercanos a la autoeficacia como la esperanza o la dureza.

Los resultados de este estudio también adicionan al conocimiento mostrando que las diferencias culturales (México versus EE.UU.) no afectan de modo significativo a la mediación de la dureza, la esperanza y la vitalidad en la relación entre la PFT y la autoeficacia. Aunque intrigante, esto quizá se deba a que simplemente las diferencias culturales entre México y EE.UU. no son tan fuertes como para amplificar (o disminuir) los efectos mediadores propuestos. Una alternativa más provocadora es que el poder de mediación de las variables estudiadas es tal que es mayormente insensible a las 
diferencias entre culturas. En cualquier evento, los hallazgos mostrados son reveladores ya que sugieren que, por lo menos en México y EE.UU., algunos de los factores que transmiten la influencia de la PFT a la autoeficacia tienen una relevancia similar.

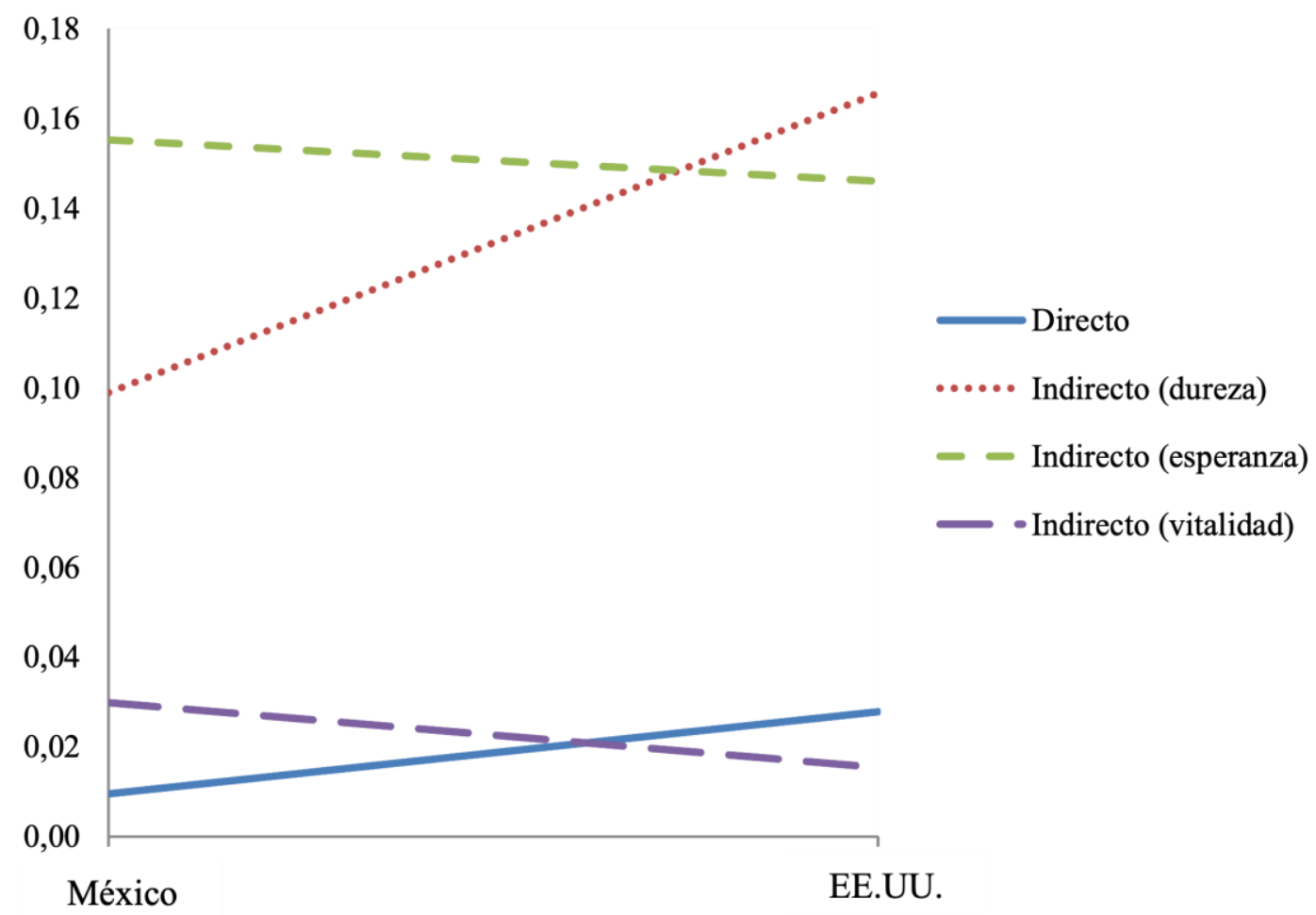

Figura 2. Comparación de los coeficientes de mediación entre México y EE.UU.

\section{Limitaciones e investigación futura}

Una limitación de este trabajo es que sólo se abordan tres mediadores y esto se hace de forma paralela. Posteriores investigaciones podrían estudiar el efecto mediador de otros factores que también fomentan la perseverancia (p.ej. optimismo) y examinar la estructura de relaciones entre los mediadores. Una alternativa es el método de mediación en serie como lo explica Hayes (2013). Otras investigaciones también pueden analizar muestras más grandes (y de varios países) para aumentar el poder estadístico de las pruebas. Resulta intrigante que, en la Figura 2, la mediación de la dureza sea bastante más pronunciada en EE.UU. que en México. Quizá un mayor poder estadístico de la prueba revelaría que esta diferencia en realidad es significativa.

\section{Conclusión}

La PFT y la autoeficacia son conceptos importantes la sociedad contemporánea. La relación entre ellos muy probablemente se transmite a través de factores que, con una 
clara orientación futura, fomentan la perseverancia y el éxito como la dureza, la esperanza y la vitalidad. El poder de mediación de estos factores es diferente pudiendo ser invariante entre México y EE.UU. Entre más se sepa sobre los mediadores de la relación entre la PFT y la autoeficacia, y su estabilidad o cambio en varios países, se podrán diseñar estrategias para convertir más eficazmente la PFT en fuertes creencias de autoeficacia que ayuden a la gente de diversas localidades a mejorar sus resultados laborales y su vida. 


\section{Referencias}

Bandura, A. (1977). Self-efficacy: Toward a unifying theory of behavioral change. Psychological Review, 34 (2), 191-252. https://doi.org/10.1037//0033295x.84.2.191

Bandura, A. \& Locke, E. (2003). Negative self-efficacy and goal effects revisited. Journal of Applied Psychology, 88 (1), 87-99. https://doi.org/10.1037/00219010.88.1.87

Bond, M. H. (1988). Finding universal dimensions of individual variation in multicultural studies of values. Journal of Personality and Social Psychology, 55 (6), 1009-1015. https://doi.org/10.1037/0022-3514.55.6.1009

Cernas-Ortiz, D.A., Mercado-Salgado, P. \& Davis, M. (2018). Perspectiva futura de tiempo, satisfacción laboral y compromiso organizacional: El efecto moderador de la autoeficacia, la esperanza y la vitalidad. Revista de Psicología del Trabajo y de las Organizaciones, 34 (1), 1-9. https://doi.org/10.5093/jwop2018a1

Cole, M., Bruch, H. \& Vogel, B. (2006). Emotion as mediators of the relations between perceived supervisor support and psychological hardiness on employee cynicism. Journal of Organizational Behavior, 27 (4), 463-484. https://doi.org/10.1002/job.381

Cole, M., Feild, H. \& Harris, S. (2004). Student learning motivation and psychological hardiness: Interactive effects on students` reactions to a management class. Academy of Management Learning and Education, 3 (1), 64-85. https://doi.org/10.5465/amle.2004.12436819

Corral-Verdugo,V., Fraijo-Sing, B. \& Pinheiro, J. (2006). Sustainable behavior and time perspective: Present, past, and future orientations and their relationship with water conservation behavior. Revista Interamericana de Psicología, 40 (2), 139-147.

Epel, E., Bandura, A. \& Zimbardo, P. (1999). Escaping hopelessness: The influences of self-efficacy and time perspective on coping with homelessness. Journal of Applied Social Psychology, 29 (3), 575-596. https://doi.org/10.1111/j.15591816.1999.tb01402.x

Hair, J., Black, W., Babin, B., Anderson, R. \& Tatham, R. (2006). Multivariate Data Analysis. Upper Saddle River, NJ: Pearson-Prentice Hall.

Hayes, A. (2013). Introduction to Mediation, Moderation, and Conditional Process Analysis: A Regression-Based Approach. New York: The Guilford Press.

Hofstede, G., Hofstede, G, J. \& Minkov, M. (2010). Cultures and Organizations. Software of the Mind. New York: McGrawHill.

Hofstede, G.J. (2015). Culture`s causes: The next challenge. Cross-cultural Management: An international Journal, 22 (4), 545-569. https://doi.org/10.1108/CCM-03-2015-0040

Kline, R. B. (2011). Principles and Practice of Structural Equation Modeling. Nueva York: Guilford Press.

Lindell, M. \& Whitney, D. (2001). Accounting for common method variance in cross-sectional research designs. Journal of Applied Psychology, 86 (1), 114121. http://dx.doi.org/10.1037/0021-9010.86.1.114

Locke, E. \& Latham, G. (2009). Has goal setting gone wild, or have its attackers abandoned good scholarship? Academy of Management Executive, 23 (1), 1723. https://doi.org/10.5465/amp.2009.37008000 
Maddi, S. (2002). On hardiness and other paths to resilience. American Psychologist, 54 (3), 175-185. https://doi.org/10.1037/0003-066x.60.3.261

Maddi, S. (2013). Turning Stressful Circumstances into Resilient Growth. New York: Springer.

Miraglia, M., Cenciotti, R., Alessandri, G. \& Borgogni, L. (2017). Translating selfefficacy in job performance over time: The role of job crafting. Human Performance, 30 (5), 254-271. https://doi.org/10.1080/08959285.2017.1373115

Navarro, J., Roe, R. A. \& Artiles, M. I. (2015). Taking time seriously: Changing practices and perspectives in work/organizational psychology. Revista de Psicología del Trabajo y de las Organizaciones, 31 (3), 135-145. https://doi.org/10.1016/j.rpto.2015.07.002

Oettingen, G. (1995). Cross-cultural perspectives on self-efficacy. En Bandura, A. (Ed.), Self-efficacy in Changing Societies, 149-176. New York: Cambridge University Press.

Park, I. \& Jung, H. (2015). Relationships among future time perspective, career and organizational commitment, occupational self-efficacy and turnover intention. Social Behavior and Personality, 43 (9), 1547-1562. https://doi.org/10.2224/sbp.2015.43.9.1547

Park, N., Peterson, C. \& Seligman, M. (2004). Strengths of character and well-being. Journal of Social and Clinical Psychology, 23 (5), 603-619. DOI: https://doi.org/10.1521/jscp.23.5.603.50748

Peterson, C., Park, N., Hall, N. \& Seligman, M. (2009). Zest and work. Journal of Organizational Behavior, 30 (2), 161-172. http://dx.doi.org/10.1002/job.584

Ryan, R. \& Frederick, C. (1997). On energy, personality, and health: Subjective vitality as a dynamic reflection of well-being, Journal of Personality, 65 (3), 529-565. https://doi.org/10.1111/j.1467-6494.1997.tb00326.x

Schyns, B. \& von Collani, G. (2002). A new occupational self-efficacy scale and its relation to personality constructs and organizational variables. European Journal of Work and Organizational Psychology, 11 (2), 219-241. https://doi.org/10.1080/13594320244000148

Seligman, M. (2006). Learned Optimism. How to Change your Mind and your Life. New York: Vintage Books.

Skomorovsky, A. \& Sudom, K. (2011). Role of hardiness in the psychological wellbeing of Canadian Forces officer candidates. Military Medicine, 176 (1), 7 12. https://doi.org/10.7205/milmed-d-10-00325

Snyder, C.R., Rand, K., King, E., Feldman, D. \& Woodward, J. (2002). "False" hope. Journal of Clinical Psychology, 58 (9), 1003-1022. https://doi.org/10.1002/jclp.10096

Snyder, C.R., Sympson, S., Ybasco, F., Borders, T., Babyak, M. \& Higgins, R. (1996). Development and validations of the state hope scale. Journal of Personality and Social Psychology, 70 (2), 321-335. https://doi.org/10.1037//0022-3514.70.2.321

Stajkovic, A., Bandura, A., Locke, E., Lee, D. \& Sergent, K. (2018). Test of three conceptual models of influence of the big five personality traits and selfefficacy on academic performance: A meta-analytic path-analysis. Personality and Individual Differences, 120 (1), 238-245. https://doi.org/10.1016/j.paid.2017.08.014

Stolarski, M., Mattews, G., Postek, S., Zimbardo, P. \& Bitner, J. (2014). How we feel is a matter of time: Relationship between time perspective and mood. 
Journal of Happiness Studies, 15 (4), 809-827.

https://doi.org/10.1007/s10902-013-9450-y

Walker, T. \& Tracey, T. (2012). The role of future time perspective in career decision making, Journal of Vocational Behavior, 81 (2), 150-158. https://doi.org/10.1016/j.jvb.2012.06.002

Weiner, B. (2010). The development of an attribution-based theory of motivation: A history of ideas. Educational Psychologist, 45 (1), 28-36. https://doi.org/10.1080/00461520903433596

Yongxing, G., Hongfei, D., Baoguo, X. \& Lei, M. (2017). Work engagement and job performance: The moderating role of perceived organizational support. Anales de Psicología, 33 (3), 708-713. https://doi.org/10.6018/analesps.33.3.238571

Zimbardo, P. \& Boyd, J. (1999). Putting time in perspective: A valid, reliable individual differences metric. Journal of Personality and Social Psychology, 77 (6), 1271-1288. https://doi.org/10.1037//0022-3514.77.6.1271 